\section{PUBERDADE DOS COELHOS DA RAÇA BRANCO DE NOVA ZELÂNDIA*}

\author{
ANTONIO PACHECO DE MACEDO \\ Medico Veteringrio, MS \\ Faculdade de Medicina Veterinaria \\ Zootecnia da USP \\ DMRR MIGUEL \\ Professor Adjunto \\ Faculdade de Medicina Veterindria e
} Zootecnia da USP

MACEDD. A.P. \& MIGUEL, O. Puberdade dos coelhos da raca Branco de Nova Zelandia. Rev.Fac. Med.vet Zootec. Univ.S.Paulo, $23(1): 55-67$
1986.

RESUMO: Estudou-se puberdade dos coelhos usando-se 41 machos oa raca Branco de Nova Zelandia, at a idade de 130 dias. O comoortamento de monta - as capacterlsticas espermaticas foram estudadas Dara a avaliacso o puberdade da maturidade sexual cos coelnos. Fol oaservado que puberdade cesses coelnos situou-se entre 72 . 126 dias a maturidade sexuab entre 98 e 125 dias de idoce.

UNITERMOS: 1. Reproducso, coelhos: 2 . Semen; 3. Puberdade; 4. Sexo, maturidadet

- Trabalho realizado com auxiluo do Conselho Nacional de Desenvolumento Científico e Tecnologico.

\section{INTRODUGAO}

Uários pesquisadores têm empregado o coelho macho em suas pesquisas, mas, nem sempre, registraram a idade na qual eles foram iniciados como doadores de sêmen. No Quad. 1 observa-se que a idade mais baixa foi de 20 semanas. R classificacro em adultos justifica-se pela producyo de espermatozbides. Ainda neste Duad.1 encontram-se os valores da Volume ( $m l$ ) e pH determinados no semen de coelhos constatando-se os valores de 0,02 a $6,00 \mathrm{ml}$ e 6,40 a 8,40 , respectivamente. 0 Ouad. 2 apresenta a Idade, volume (ml), fraço llquida ( $m l$ ), fracgo gelatinosa $(\mathrm{ml})$ e fracăo gelatinosa (\$), que variaran de $11,52 \%$ a 75,40\%. D Ouad. 3 discrimina as várias concentracoles em espermatozbides do sêmen de coelho, as quais tiveram una variacao de 57 a $2.700 \times$ $10^{3} / \mathrm{mm}^{3}\left(\times 10^{3}\right)$. 0 Duad. 4 registra os valores determinados no estudo das alteracoles da morfologia espermatica do coelho, que variaram de $4,15 \%$ a $37,96 \%$.

A puberdade, segundo consideraram DONOUAN \& WERFF TEN BOSCH, J.J., 11 (1965), e un estagio no qual a funcajo endocrina dos testiculos torna-se evidente, os estudos de SKINNER, 44 (1967), proporcionam a observacro de que o intcio da puberdade coincide com ocasia em que os testlculos iniciam sua atividade androgenica $a$ animal adquire caracterlsticas de comportamento de macho. Observou, ainda, que aos 63 dias de idade os testiculos tinham descido para dentro do escroto, aumentaram de modo acelerado e o comportamento agressivo tornouse mais pronunciado. Os resultados encontrados por este pesquisador tambem estao de acordo com as ooservacóes de DAVIES \& MANN, 9. (1947), que mostram que a atividade androgenica precede a evidencia histologica do intcio da espermatogénese. CHUBB et alii, 7 (1975) demonstraram que a testosterona cai para nlveis nao detectaveis ou desprezivejs no primejro mes apos o nascimento, com significante aumento com seis semanas de idade.

o objetivo do presente estudo é o de determinar a idade em que os coelhos da raca Branco de Nova Zelandia atingem a puberdade e em seguida á maturidade sexual, complementando-se com o estudo de algumas caracterlsticas do semen.

\section{MATERIAL E METODOS}

Foram empregados neste trabalho 41 coelhos machos, da raca Branco de Nova Zelandia (BNZ), oriundos de criacăo propria, tatuados por ocasia da desmama aos 30 dias de idade e colocados em gaiola de arame galvanizaoo com 1,50m $\times 1,20 \mathrm{~m}$, onde foj observada a mudanca oe comportamento para agressivo e inlcio de briga entre eles. Ros 65 cias de idade foram transteri- 
dos para gaiolas individuais com $0,80 \mathrm{~m} x$ $0,60 \mathrm{~m}$, tambem de arame galvanizado. Aos 70 dias foi iniciada a observaço face a uma femea colocada em sua gaiola. Desde o pri: meiro salto foi procedida a coleta de semen com vagina artificial, segundo MACEDO, 34, 1979, diariamente, e determinado: o volume diretamente no tubo coletor e a fraço liquida, apos o desearte da fraca gelatinosa, se presente, segundo MACIRONE \& WAL TON, 36, 1938, e, em seguida, a fraço gelatinosa por oiferenca; o pH com papel indicador especial (Neutralit, Merek, Darmstad) nos (imites de $5.5 \longmapsto 5,00$, com escala em cores; concentracaro em espermatozóides $/ \mathrm{mm}^{3}$, em cgmara de Neubauer; a morfologia espermatica em esfregacos corados pelo método de UILLIRMS, 47, 1920, modificado por LAGERLöf, 31, 1934; foi calculada a concentraczo em espermatozodides/ejaculado. Foram realizadas, durante - trabalno, 1.156 coletas de semen e 463 naro foram conseguidas. As coletas arolonga$r a m-s e$ ate 130 dias de idade. Os coelhos foram pesados no dia em que forneceram o 10 ejaculado, o lo ejaculado com espermatozoides e aos 130 dias de idade. Foram alimentados com água e raço Coelhil "R" * : Umidade (maximo) 14,05\%; Protelna bruta (minimo) 17.00\%; Extrato etéreo (mfnimo) 2,00\%; Materia fibrosa (maximo) 16,00\%; Materia mineral (maximo) 11x; Calcio (ma$x$ imo) 2,50\%; Fosforo (m1nimo) 0,42\%.

\section{RNALISE ESTATISTIEA}

Foram calculados, segundo GDLDSTEIN 19, 1965, os valores da média aritmética desvio padra, coeficiente da variabilidade de Pearson e a mediana, bem como o coeficiente de correlacáo de Pearson e a regress go linear entre as varias variaveis em estudo. Estes coeficientes foram testados. utilizando-se o teste" $t$ " de 5tudent.

\section{RESULTADOS}

A atitude agressiva entre os coelhos foi observada a partir dos 60 dias de idade tendo esse comportamento de macho aumentado progressivamente.

Ds resultados obtidos neste trabalho estgo incluldos nas Tab. 1,2, e 3. A Tab. 1 apresenta os valopes da media aritmética, desvio padra, coeficiente de variabilidade de Pearson e a mediana encontrados para as variáveis em estudo. As Tab. 2 e 3 registram os valores da Regressá Linear, Coeficiente de Correlacazo de Pearson ( $r$ ) e o teste " $t$ " de Student das varias caracteristicas estudadas.
O primeiro ejaculado foi obtido aos 72 dias de idade em 1 coetho $(2,44 x)$; entre 72 e 77 dias em 5 coelhos $(12,20 \%)$; entre 72 e B4 dias em 18 coelhos $(43,90 \%) ;$ entre 72 e 90 dias em 24 coelhos $(58,54 \%)$; entre 72 e 98 dias em 31 coelhos $(75,61 \%)$; entre 72 e 106 dias em 39 coelhos $(95,12 \%)$ e entre 72 e 126 dias em 41 coelhos (100\%).

- primeiro ejaculado com espermato$z$ oides foi obtido aos 98 dias de idade em 1 coetho $(2,44 \%)$; entre 98 e 103 dias em 8 coethos $(19,51 \%)$; entre 58 e 108 dias em 19 coethos ( $46,34 \%)$; entre 98 e $113 \mathrm{dias}$ em 29 coelhos $(70,73 \%)$; entre 98 e 120 dias em 34 coelhos ( $82,93 \%)$; entre 98 e 127 dias em 39 coelhos $(95,12 \%)$ e entre 98 e 129 dias em 41 coelhos $(100 \%)$.

O intervalo medio entre o 10 ejaculado - 10 ejaculado com espermatozbides foi de $21,30 \pm 10,40(1 \longmapsto 37)$ dias com a mediana de 24 dias.

0 levantamento da patología espermatica revelou que as formas patologicas atingiram $30 \%$ aos 98 100 dias de idade diminuindo para $15,60 \%$ aos 126 dias. As formas normais que inicialmente, oos $98 \longmapsto 100$ dias, eram de 69,90\%, aumentaram para $84,20 \%$ aos $126 \longmapsto 130$ dias de idade.

A fraço gelatinosa foi detectada em 486 ejaculados $(41,90 \%)$. Ela representa 57,6593\% do volume total com espermatozoides.

Pela andise da Tab. 9 verifica-se que :

a) o $\mathrm{pH}$ encontrado neste estudo foi de $7,60 \pm 0,60(6,90 \longmapsto 8,50)$ para os ejaculados sem espermatozoides e 7,49 $\pm 0,32(6,68 \longmapsto 8,00)$ para os ejacuiados com espermatozoides. Um coelho forneceu semen ligeiramente acido, tanto nos ejaculados sem espermatozoides $(n=6)$ como nos ejaculados com espermatozbides $(n=18)$ fixando-se em $6,92 \pm 0,18$ e $6,90 \pm 0,31$, respectivamente, mantendo-se, entretanto, bem proximo da normalidade:

b) o volume total $(\mathrm{ml})$ encontrado foi de $0,43 \pm 0,26(0,14 \longmapsto 1,50)$ para os ejaculados sem espermatozoides e de $0,65+0,22(0,27 \longmapsto 1,36)$ para os ejaculádos com espermatozdides;

c) a concentracăo em espermatozódes $/ \mathrm{mm}^{3}$ foi de $40.223 \pm 26.424$ (885 107.738 ) e a concentracro em espermatozoideslejaculado $\left(\times 10^{3}\right)$ foi de $17.697 \pm 15.939(339 \longmapsto 48.677)$.

A andise das Tab. 2 e 3 mostra que o Coeficiente de Correlaca de Pearson apresenta uma correlaço positiva de média intensidade e significente entre: Peso no 
10 ejaculado e o Voiume $(r=0,47)$, Peso no 10 ejaculado e Peso aos 130 dias de idade $(r=0,44)$, Peso 10 ejaculado com espermatozóides e Concentraca em espermatozojdes $/ \mathrm{mm}^{3}$ ( $\left.r=0,52\right)$; uma correlaça positiva de alta intensidade e significante entre o Peso no 10 ejaculado e Peso no 10 ejaculado com espermatozoides $(r=0,70)$. Revela, tambem, uma correlaczo de baixa intensidade $e$ na siginificante quando foram correlacionadas as demais caracteristicas.

\section{DISCUSSAO}

A andise das caracterlsticas fisicas de um semen, segundo LAGERLöf, 31, 1934, procedida atraves das observacás macro e microscopicas, juntamente com outros dados semiologicos, conduz a uma avaliacaro do estado reprodutivo de um macho. Em conseqoencia, realizamos o levantamento de algumas caracterlsticas como elementos complementares ao presente estudo.

0 volume total $(\mathrm{ml})$ determinado para os ejaculados com espermatozdides de 0,65 t $0,22(0,27-1,36)$ está proximo e acima do encontrado por GREGOIRE et alii, 22 , $1956(0,59)$ e proximo e abaixo do de GOTZE, 21, $1949(0,70)$. Os limites estyo compreendidos entre o proximo e acima do de O'FER RAL \& MEACHAN, 49, $1968(0,15)$ e proximo e abaixo co de MACÉDo et alii, 35, 1982 $(1,40)$ (Quad. 1).

- DH determinado para os ejaculados com espermatozbides ce $7,49 \pm 0,32 \quad 0,68$ 8,00) esta proximo e acimado encontrado por MACARI et a tij, 32, 1977, de $7,43(7,22 \longmapsto 7,76)$ e proximo e abaixo do de O'FERRAL \& MERCHAN, 41, 1968, de 7,59 $(6,45 \longmapsto 8,40)$. Os limites encontram-se tambem compreendidos entre o proximo e acima determinado por WHITE, 46, 1958 $(6,60)$ e do mesmo valor $(8,00)$ encontrados por MACEDD et alij, 35, 1982. (Quad. 1).

A Praczo gelatinosa encontrada $57,65 \%$ achia-se compreendida entre as deter. minadas por DUEIEL, $12,1973(43,33 \%)$ e a de GROVE, 23, 1963 (64,05\%). (Quad. 2 ).

A concentraczo em espermatozoides $/ \mathrm{mm}^{3}$ encontrada de $40.223(885$ 107.738) nao pode ser comparada com os resultados determinados por varios pesquisadores ( 0 uad. 3 ), tendo-se em vista a grande diferenca de idade e de peso entre os coelhos dos experimentos desenvolvidos e dos encontrados neste trabatho.

- bevantamento da patologia espermatica aos 126 - 130 dias de idade em $15,60 \%$, situou-se proximo dos resultados encontrados por SCHULZE, 43, 1964 ( $Q_{L}$ ad. 4).

A literatura consultada ná registra nenhum estuco relacionando o peso dos animais em suas vapias idades com outras va$r$ iáreis como o volume, $\mathrm{DH}$, concentraça em espermatozoides $/ \mathrm{mm}^{3}$ e concentraca em es-
Dermatozbideslejaculado, o que roi reali$z$ ado neste trabatho.

\section{CONCLUSOES}

Tendo em vista as condicoses estabelecidas para o presente experimento, os resultados obtidos, analisados e discutidos, parece-nos licito concluir que:

1. o primeiro ejaculado dos coelhos da raca BNZ pode ser obtido a partir de 72 dias de idade e com 126 já se obtem em todos os reprodutores do plantel;

2. o primeiro ejaculado com espermatozoides pode ser obtido a partir de 98 dias de idade e aos 129 dias jà se obtem em 100\% dos reprodutores;

3. o intervalo entre o primeiro ejaculado e o primeiro ejaculado com espermatozodides foi de $21 \pm 10,40$ ( dias, com a mediana de $24 \mathrm{dias}$

4. O pH bixou com o aumentio da amostra e, portanto, com o aumento da idade dos coelhos, tanto nos valores medios globais como nos valores individuais dos ejaculados sem e com espermatozoides. $D \mathrm{pH}$ manteve-se proximo da neutralidade ou ligeiramente alcalino;

5. o volume $(m l)$ dos ejaculados aumentou em seus valores medios glooais e valores medios individuais com o aumento da amostrae, portanto, com o aumento da idade dos coelhos:

6. a patogia espermatica no periodo de 98 a 100 dias alcancou $30 \%$ e diminuiu. gradativamente, para a metade-15,60\% aos $126 \longmapsto 130$ dias de idade. concorrendo para o aumento das formas normais de $60,90 \%$ para 84,20\% nos mesmos periodos;

7. existe uma correlacăo positiva de média intensidade e significante entre: peso no 10 ejaculado e volume, peso no 10 ejaculado e peso aos 130 dias de idade, Deso no 10 ejaculado com espermatozoides e concentraczo em espermatozbides $/ \mathrm{mm}^{3}$; uma correlacăo positiva e de alta intensidade e significante entre: Deso no 10 ejaculado e peso no 10 ejaculado com espermatozóides. Com relacaro as demais caracterlsticas estudadas verificou-se haver uma correlaca de bajxa intensidade e nro significante:

8. com base nestes resultados as coelhos da Paca Sranco de Nova Zelandia atingem a puberdade com 72 a 125 dias e a maturidade sexual com 98 a 125 cias de idade. 
MACEDO, R.P. \& MIGUEL, O. Puberty of New Zealand white raboits. Rev.Fac.Med.vet.Zootec.Univ. 5.Paulo, $23(1): 55-67,1986$.

SUMMARY: The puberty of rabits were studied using 49 males Neu Zealand wites, until the age of 130 days. Mountings behabiour and the spermatic characteristics vere studied for evaluation of their puberty and sexual maturity. It was observed that the puberty in those rabuits was room 72 to 126 days and the sexual maturity, from 98 ta 129 days of age.

UNITERMS: 1. Reproduction of paboltsit ${ }^{+}$. Semeni $i^{+}$ 3. Puberty; 4. Sexual maturity ${ }^{+}$

QUADRD i - Idade, volume e pH determinados em amostras de semen de coelho, segundo varios pesquisadores. Sro Paulo, 1985.

\begin{tabular}{|c|c|c|c|}
\hline $\begin{array}{c}\text { Aruce } \\
\text { ce } \\
\text { ordex }\end{array}$ & $\therefore L=0 R \equiv S$ & Volume (ml) & $\mathrm{pH}$ \\
\hline 1 & MACIRONE, $C$ \&ALTON, $\wedge^{36}$ (1938) $\ldots \ldots \ldots \ldots$ adultos & 0,32 & - \\
\hline 2 & EDHíRSS, ?. J., $1940^{15} \ldots \ldots \ldots \ldots \ldots \ldots \ldots$ adultos & 1,99 & - \\
\hline 3 & Enex:s, c. h., $194 \gamma^{-16} \ldots \ldots \ldots \ldots \ldots \ldots \ldots$ adulcos & & $7,66: 7,00 \mapsto 8,20)$ \\
\hline 4 & $G 5: z \equiv, P . \therefore, 1949^{2}: \ldots \ldots \ldots \ldots \ldots \ldots \ldots \ldots$ adultoos & $0,70(0,60 \longmapsto 2,00)$ & - \\
\hline 5 & Eiss, $J \ldots 1952^{25} \ldots \ldots \ldots \ldots \ldots \ldots \ldots \ldots \ldots$ adultos & $0,97(0,55-1,61)$ & $6,58(6,50-7,05)$ \\
\hline 6 & 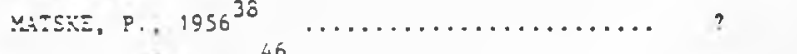 & $1,00(0,30 \longmapsto 1,60)$ & - \\
\hline 7 & WHETE, I. C., $1958^{40} \quad \ldots \ldots \ldots \ldots \ldots \ldots \ldots \ldots \ldots$ aduleos & $1,00(0,40 \longmapsto 6,00)$ & $7,05(6,60 \mapsto 7,50)$ \\
\hline 8 & 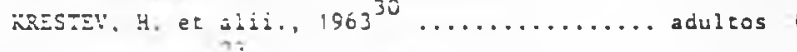 & $0,80(0,15 \mapsto 2,60)$ & - \\
\hline 9 & GRo: $:, J ., 1963^{-3} \ldots \ldots \ldots \ldots \ldots \ldots \ldots \ldots \ldots$ adulicos & $0,50(0,02 \curvearrowleft 2,62)$ & - \\
\hline 10 & 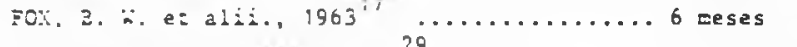 & $(0,10 \mapsto 0,90)$ & - \\
\hline il & 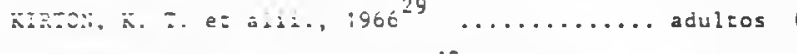 & $0,54 \pm 0,04$ & $6,80=0,64$ \\
\hline 12 & 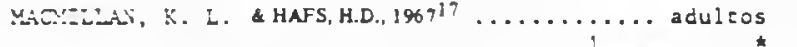 & 0,91 & - \\
\hline 13 & ANAN, R. P. \& LAYEIASE Jor., J. T., $1967^{1}$... 20 sez." & 0,50 & - \\
\hline 14 & 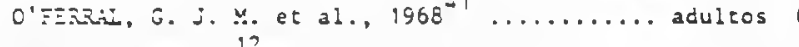 & $0,49(0,15 \longmapsto 1,05)$ & $7,59(6,45 \longmapsto 8,40)$ \\
\hline 15 & $\Sigma=\Sigma \Sigma$, s., $197 j^{12}, \ldots \ldots \ldots \ldots \ldots \ldots \ldots \ldots \ldots$ adultos & $1,55(0,47 \mapsto 5,00)$ & - \\
\hline 16 & 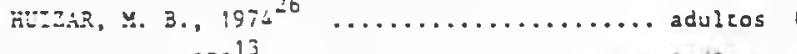 & $0,68(0,49-0,94)$ & - \\
\hline 17 & DUE:EL, s., $: 975^{13} \ldots \ldots \ldots \ldots \ldots \ldots \ldots \ldots \ldots 6 / 3 \mathrm{a}$ & $1,55(0,47-5,00)$ & - \\
\hline 18 & 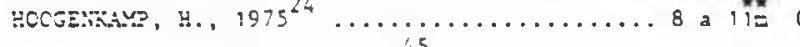 & $0,58(0,40 \longmapsto 0,80)$ & $7,22(7,02 \ldots 7,37)$ \\
\hline 19 & hE:ZzE, K. E. et adii., $1976^{42}, \ldots \ldots \ldots \ldots \ldots$ aduleos & $0,42(0,28 \sim 0,56)$ & $7,42(7,26 \mapsto 7,62)$ \\
\hline 20 & r.venEE:A, $\because$. 5. \& NOROZCVA, K. X., $1978^{2}$... adulcos & $0.80(0,30 \longmapsto 2,00)$ & - \\
\hline 21 & 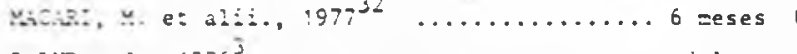 & $0,79(0,64 \longmapsto 1,08)$ & $7,43(7,22-7,76)$ \\
\hline 22 & 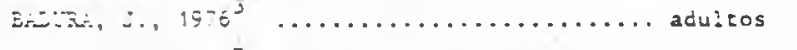 & 0,72 & - \\
\hline 23 & 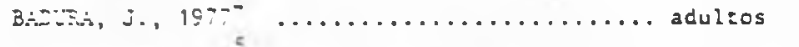 & 1,20 (Esasāo liquida) & - \\
\hline 24 & Entons, J., $1978^{5} \quad \ldots \ldots \ldots \ldots \ldots \ldots \ldots \ldots \ldots$ adultos & 1,10 (fraçāo liquida) & - \\
\hline 23 & 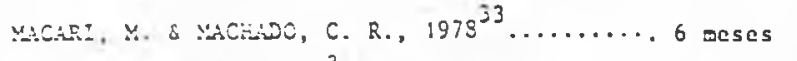 & $0,73(0,44-1,10)$ & $7,17(6,90 \longmapsto 7,60)$ \\
\hline$=\dot{9}$ & $\because$ icsso, $A . ?$ ce, $1979^{34} \ldots \ldots \ldots \ldots \ldots \ldots$ zeses & $0,74(0,30 \longmapsto 1,40)$ & $7,74(6,40 \mapsto 8,00)$ \\
\hline$\because$ & 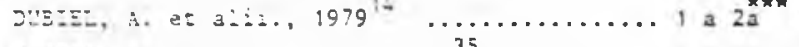 & 0,97 (fraça liquida) & - \\
\hline$\vdots \vdots$ & MCEso, $\ldots$. ?. aE et $a: i i ., 1982^{35} \ldots \ldots \ldots \ldots 6$ Deses & $0,74(0,30 \curvearrowleft 1,40)$ & $6,74(6,40 \longmapsto 8,00)$ \\
\hline
\end{tabular}

nevias $\quad * *$ ases $\quad * * *$ anos 

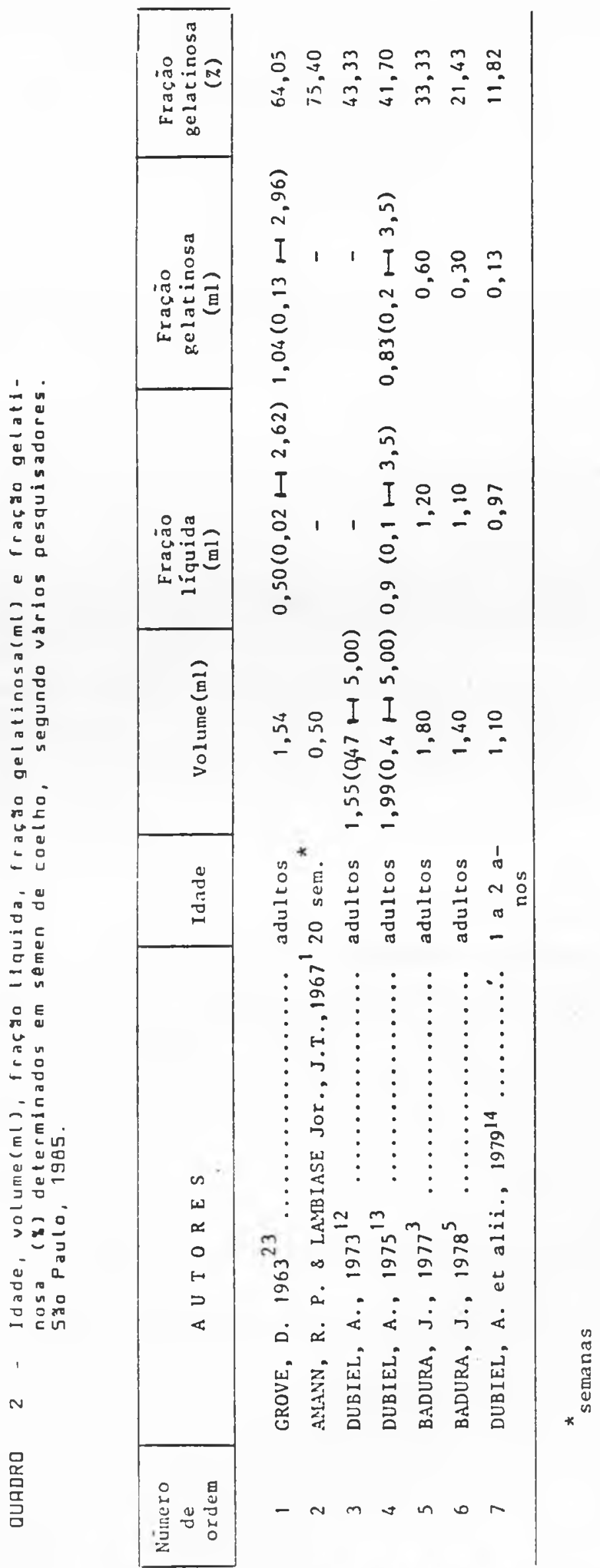

Rev.Fac.Med.vet.Zootec.Univ.S.P̃aulo, 23(1):55-67, 1986. REFERE IICIA 


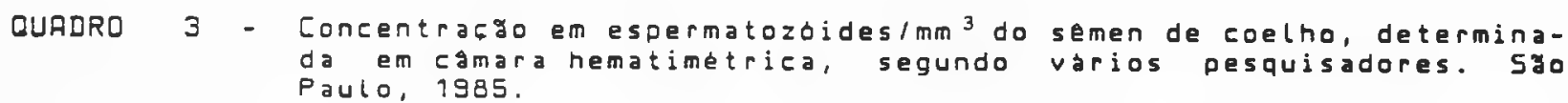

\begin{tabular}{|c|c|c|}
\hline $\begin{array}{l}\text { Nümero } \\
\text { de } \\
\text { ordem }\end{array}$ & $A U T O R E S$ & $\begin{array}{l}\text { Concentraçäo em } \\
\text { espermarozoides/min' (x103) }\end{array}$ \\
\hline 1 & 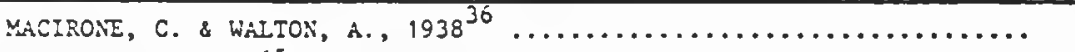 & 200 \\
\hline 2 & 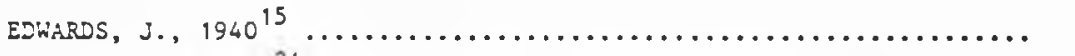 & 360,630 \\
\hline 3 & 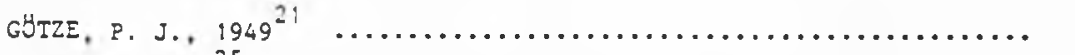 & $(0,1 \mapsto 2,0)$ \\
\hline 4 & 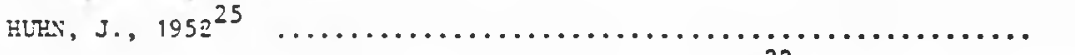 & $1.470(440 \curvearrowleft 2.700)$ \\
\hline 5 & GREGOIRE, A. T.; BRATTON, R. W.; FOOTE, R. H., $1956^{22} \ldots \ldots \ldots \ldots \ldots$ & $376(317 \mapsto 407)$ \\
\hline 6 & 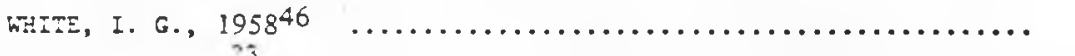 & $700(100-2.000)$ \\
\hline 7 & 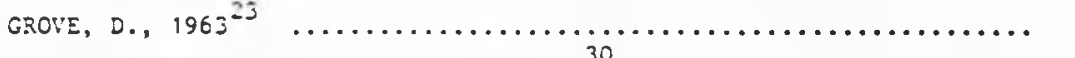 & $263,366(1,2-985)$ \\
\hline 8 & 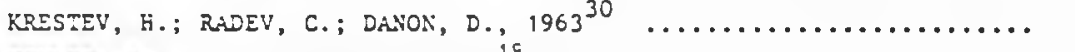 & $534(182-959)$ \\
\hline 9 & 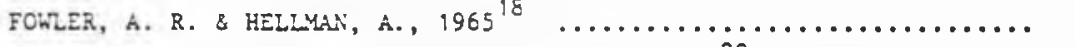 & $763(90 \sqcap 1.920)$ \\
\hline 10 & 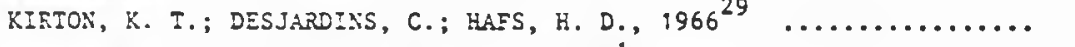 & $508 \pm 60,5$ \\
\hline 11 & 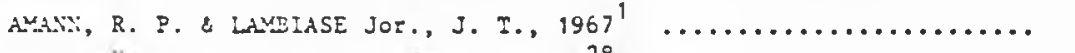 & $322 \pm 52(162 \longmapsto 752)$ \\
\hline 12 & XILHSTRÖM, ¿. E. \& FJELLSTRbM, D., $1967^{28} \ldots \ldots \ldots \ldots \ldots \ldots \ldots \ldots$ & 206,1 \\
\hline 13 & CHIANG, H. S.; IAI, Y. T.; DEL FAVERO, J. E., $1968^{8} \ldots \ldots \ldots \ldots \ldots$ & $452(368 \mapsto 680)$ \\
\hline 14 & 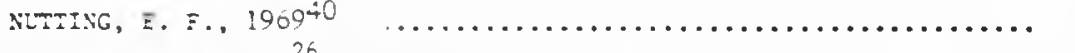 & 460 \\
\hline 15 & 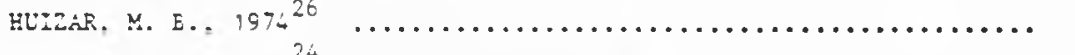 & $225(125 \curvearrowleft 361)$ \\
\hline 16 & 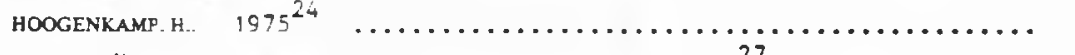 & $465,428(132 \curvearrowleft 876)$ \\
\hline 17 & 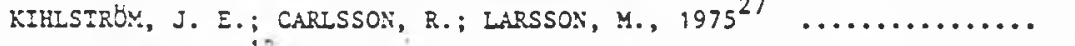 & 403,8 \\
\hline 18 & 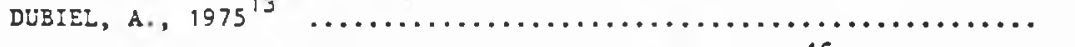 & $340(57 \curvearrowleft 1.182)$ \\
\hline 19 & WEITZE, K. F.; NthLLER-HOLTKALP, P.; STEPHAN, E., $1976^{45} \ldots \ldots \ldots \ldots$ & $272,8(154-1398)$ \\
\hline 20 & $\begin{array}{l}\text { MACARI, M.; MACHADO, C. R.; DONINI, C. A.; GARCIA, J. M.; MARCHIORI, } \\
\text { A. I.; FAGLIARI, J. J.; CARREGÉL, R. D.; SOARES, C. C., } 1977^{32} \ldots \ldots\end{array}$ & $771,8(120 \curvearrowleft 2.380)$ \\
\hline 21 & 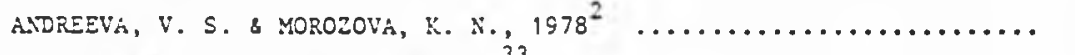 & $358(224 \curvearrowleft 454)$ \\
\hline 22 & 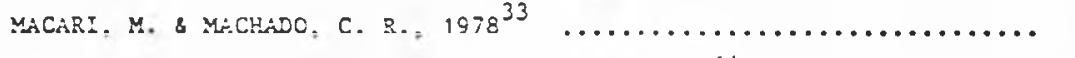 & $548,25(271 \mapsto 1.152)$ \\
\hline 23 & DUBIEL. A.: KROIINSKY, j.; KARPIAKOW'A, C., $1979^{14} \ldots$ & 97,6 \\
\hline 24 & MACEDO, A. P. de, $1979^{34} \ldots \ldots \ldots \ldots \ldots \ldots \ldots \ldots$ & $312,945(10,4 \curvearrowleft 1007,6)$ \\
\hline 25 & $\ldots \ldots \ldots \ldots \ldots \ldots \ldots \ldots \ldots \ldots \ldots \ldots \ldots \ldots \ldots \ldots \ldots \ldots \ldots \ldots$ & 77 \\
\hline 26 & MACEDO, A. P. de; MICUE:, O.; MLCCIOLO, R. C.; BARNABE, R. C., $1982^{35}$. & $312,945(10,4 \mapsto 1007,6)$ \\
\hline 27 & 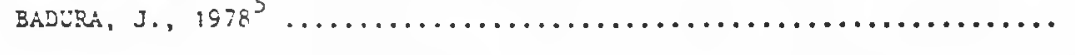 & 43,2 \\
\hline
\end{tabular}




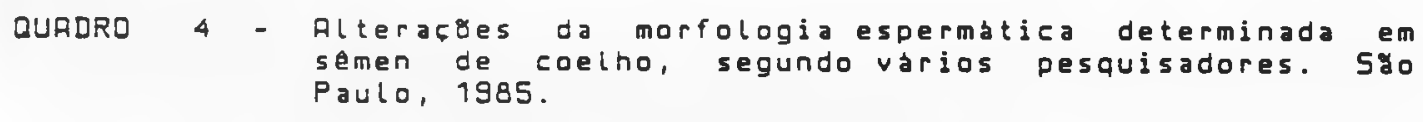

\begin{tabular}{|c|c|c|}
\hline $\begin{array}{l}\text { Numero } \\
\text { de } \\
\text { ordem }\end{array}$ & $A \cup T O R E S$ & $\begin{array}{c}\text { Altcracōes da } \\
\text { morfologia espermät } \\
\text { ca }(\%)\end{array}$ \\
\hline 1 & SCHULZE, K., $1964^{43} \ldots \ldots \ldots \ldots$ & $16,10(10,77 \longmapsto 21,72)$ \\
\hline 2 & PAUFLER, S., $1968^{42} \ldots \ldots \ldots \ldots$ & 23 \\
\hline 3 & HUIZAR, M. B., $1974^{26} \ldots \ldots \ldots \ldots$ & $16,86(9,6 \longmapsto 37,9)$ \\
\hline 4 & HOOGENKAMP, H., $1975^{24} \ldots \ldots \ldots$ & $21,20(13,9 \longmapsto 36,9)$ \\
\hline 5 & BARNABE, W. H., $1975^{6} \ldots \ldots \ldots \ldots$ & $6,442(4,15 \longmapsto 12,05)$ \\
\hline 6 & MACEDO, A. P. de, $1979^{34} \ldots \ldots \ldots$ & $20,73(8,01 \mapsto 32,89)$ \\
\hline 7 & MACEDO, A. P. de et alii., $1982^{35}$. & $20,73(8,01 \longmapsto 32,89)$ \\
\hline
\end{tabular}




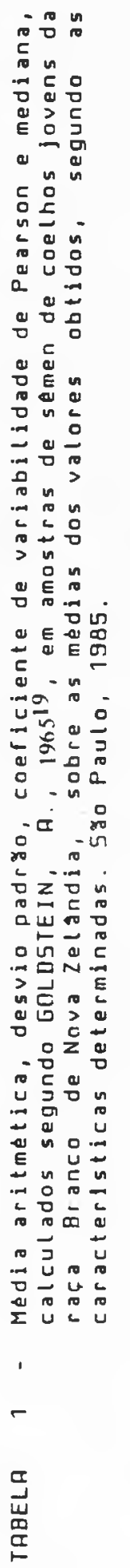

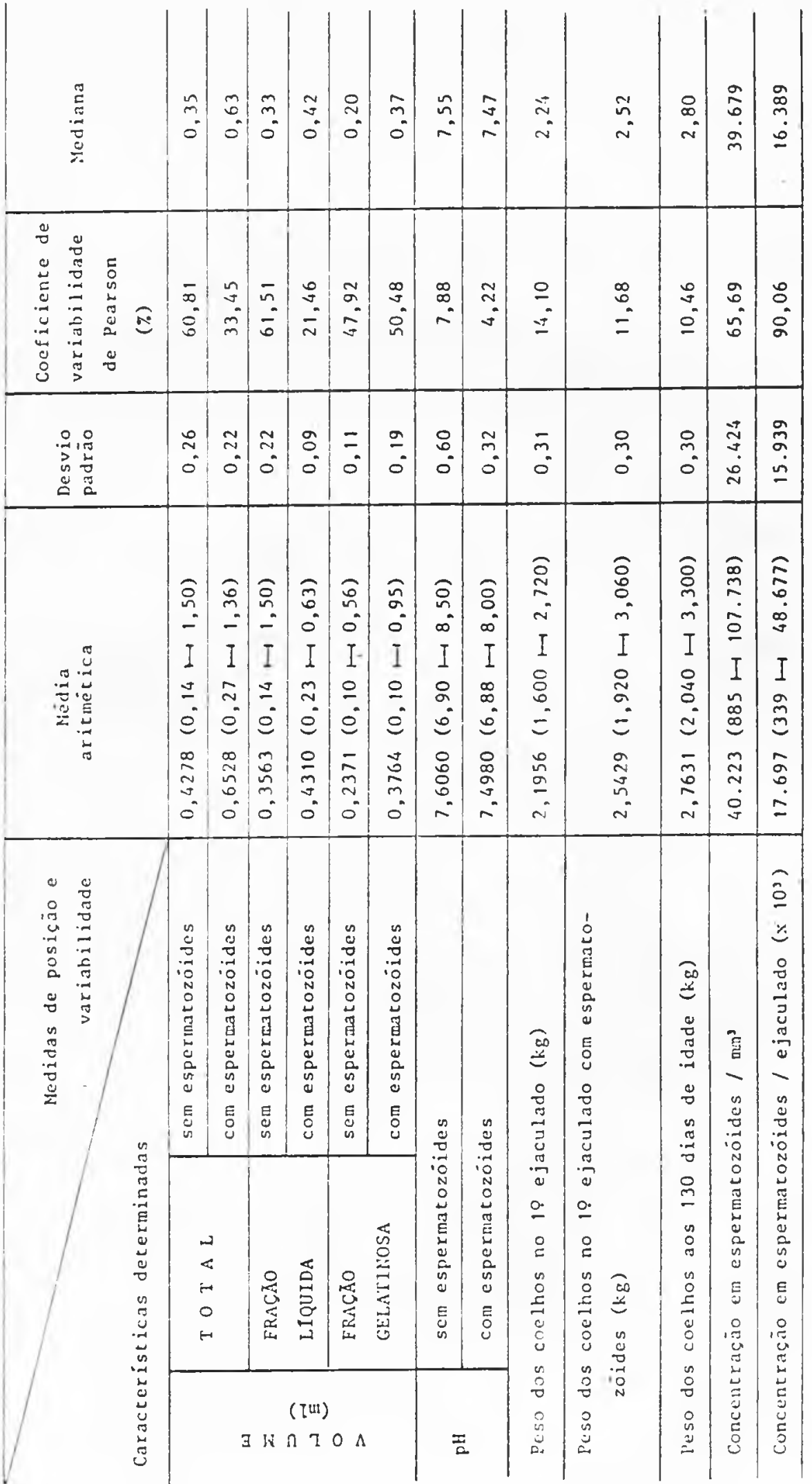




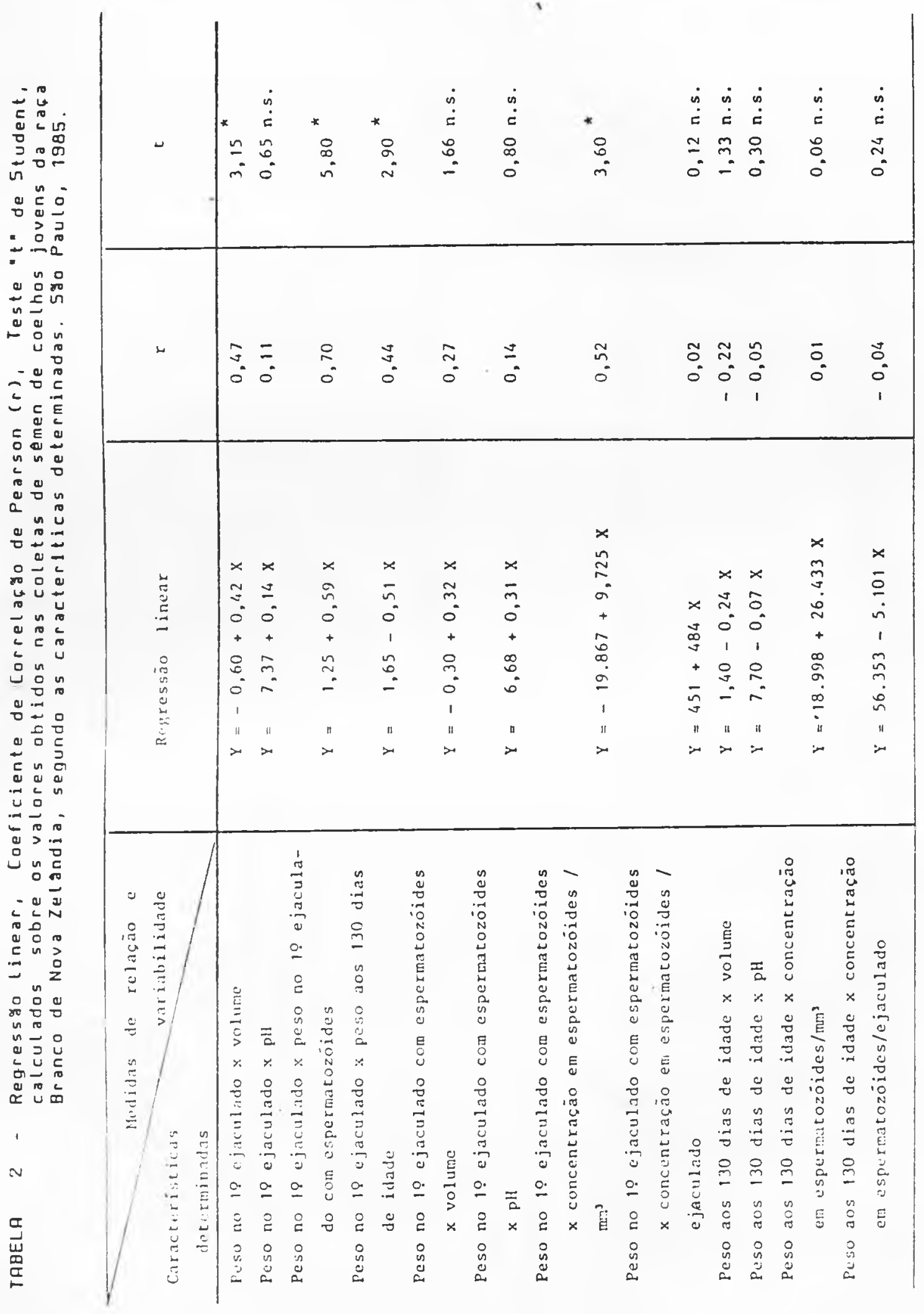




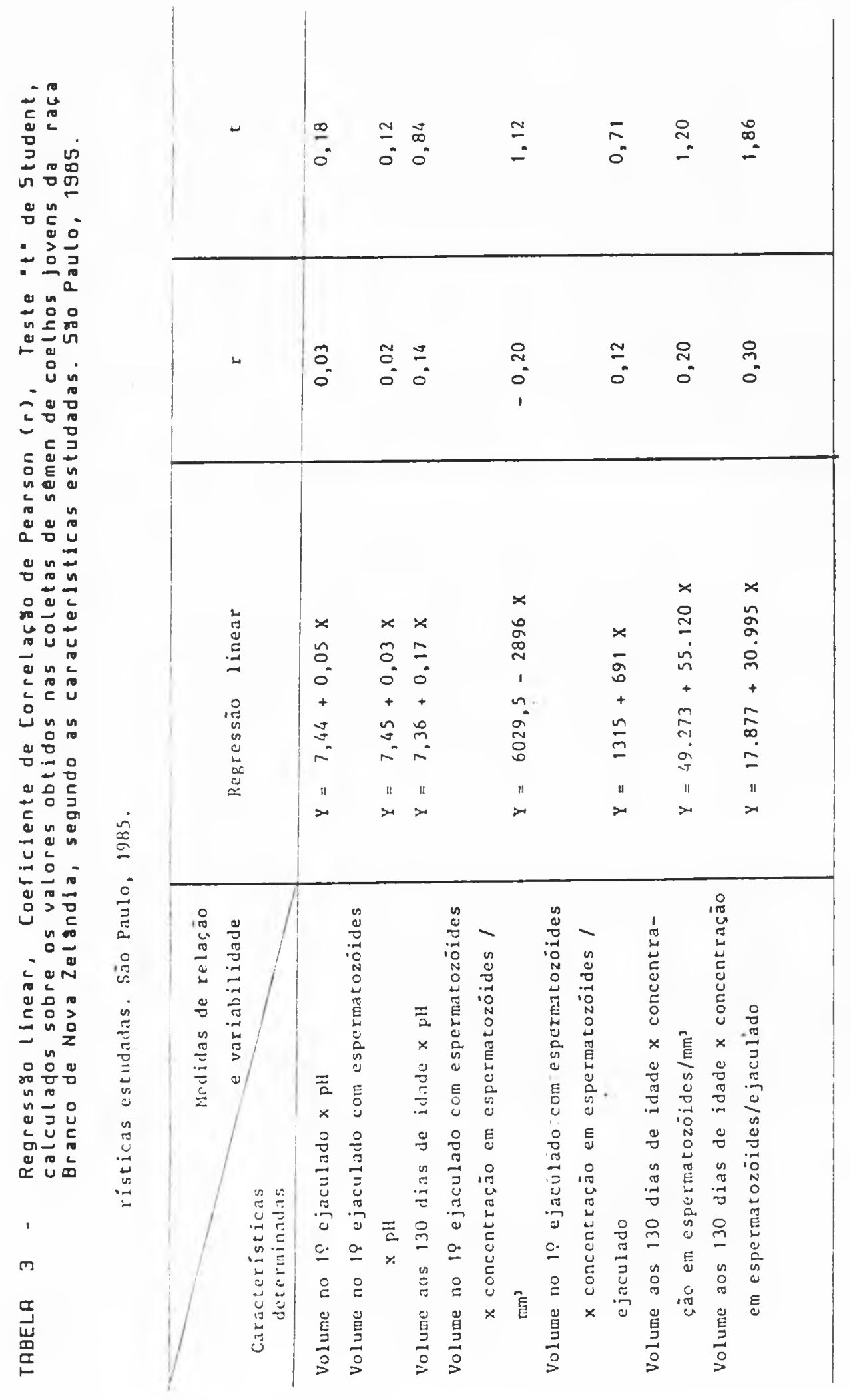


1 - RMANN, R.P. \& LAMB!ASE JR., J.T. The male rabbit. I. Changes in semen characteristics and sperm output between Duberty and one year of age. J. Reprod. Fertil., 14: 329332,1957

2 - AndReEva, U.S. \& MOROZOUA, K.N. Vitamny $\checkmark$ ratsionakh samtsov. lvitamins in rations for rabbitl. Krolikov Zverov. (5):20, 1977 apud Anim. Breed. Abstrat., 46:4065, 1978.

3 - BADURA, J. Biochemical differences in rabbit seminal plasma. In: INTERNATIONAL CONGRESS ON ANIMAL REPRODUETION AND RRTIFICIAL INSEMINATION, 8., KrakOW, 1976. Communication Abstracts. v.1., p.17 apud Anim Breed. Abstr., 45:910, 1976 .

4 - BFDURA, J. Uproszezony model sztueznej poehwy dla usyskivania nasienia oo samcow krolika. IA simplified artificial vagina for collecting semen from rabbitsl. Med. Weteryn., 33:505-606, 1977.

5 - BRDURA, J. Roznice rasowe w produkeji nasienia kroljkdw. /Breed differences in semen production of rabuitsl. Med. Weteryn., 34:229231,1978 .

6 - barnabe, V.h. Irradiaca de coelhos - Dryctolagus cuniculus (Linnaeus, 1758). Efeitos sobre a morfologia espermatica. Sro Pauto, 1975, 78p. Tiese de doutoramento - Instituto de Biociancias da Universidade de 5\%ั0 Paulo)

7 - CHUBB, [.; EWING, L.; IRBY, D. The ontogeny of testosterone (T), DHT, $3 \alpha-D I D$ and $3 \beta$-DIOL secretion by perfused rabbit testes-epidicymides. In: RNNURL MEETING OF THE SOEIETY OF THE STUDY ON REPRODUCTION, 6., 1975. Proceedings. p. 44 apud GONDOS, $B$. et alii, 20 , p. 171 .

8 - CHIANG, H.S.; LAI, M.T.; DEL FAUERO, J.E. Artificial insemination in rabbits. J. Taiwan Ass. anim. Husb. vet. Med., 13:17-23, 1958.
9 - DAUIES, D.U. \& MANN, T. Functional development of accessory glands and spermatogenesis. Nature, London, 160:295, 1947 apud SKINNER, J.D. $44,1967$.

10 - DoggetT, U.C. Periodicity in the fecundity of male rabuits. Amer. J. Physiol., 187:445-450, 1956 .

11 - DONOUAN, B.T. a van der WERF TEN 8OSH, J.J. Physiology of puberty. London, Edward Arnold. 1965 apud SKINNER, J.D. 44, 1967.

12 - DUBIEL, $A$. Dbserwacje nad uzyskiuaniem nasienia od kedlikbw. lobservations on semen collection from rabbitsl. Med. Veteryn., $29: 624-626,1973$.

13 - DUBIEL, A. Dcena wlasciwosci nasienia krolikow z uwgledinieniem zaburzen plodndsci. lEvaluation of rabbit semen characters in relation to fertility disordersl Pol. Arch. Weteryn.. 17:707-717, 1975 .

14 - DUBIEL, R.; KROLINSKI, J.; KARPIAKDWA, [. Wlasciwosci nasienia krolikow wybranych ras. ISemen characters in different. rabbit breeds/. Med. Weteryn., 35:175-178, 1979.

15 - EDWRRDS, J. The effect of unilateral castration on spermatogenesis. Proc. roy. Soc. B., 128:407-421, 1940 .

16 - EMMENS, C.W. The motility and viability of rabbit spermatozoa at different hydrogen-ion concentrations. J. Physiol., London, 106:471-481, 1947.

17 - FOX, B.W.; JACKSON, H.; CRAIG, A.W.; GLDUER, T.D. Effects of alkylanting agents on spermatagenesis in the rabbit. J. Reprod. Fertil., 5:13-22, 1963.

18 - FOWLER, A.K. \& HELLMAN, A. An eletronic method for counting sizing rabbit spermatozoa. Fertil and Steril., 16:778-784, 1985.

19 - GoLdTEIN, A. Biostatistic: an introductary text. 2.ed. New York, MeMillan, 1955. 
20 - GDNDOS, B.; RENSTON, R.H.; GDLDSTEIN D.A. Postnatal differentiation of Leydig cells in the rabbit testis. Amer.J. Anat., 145:167-182, 1976.

21 - GöTZE, $R$. Besamung und Unfruchtbarkein der Haussaugetiere. I Insemination and sterility of domestic animalsl. Kaninchen, p.548. Hannover Schaper Verlag, 1949 aDUd HOOGENKAMP, H., 24 , 1975. p.593.

22 - GREGOIRE, A.T.; BRATTON, R.W.; FOOTE, R.H. Sperm output of rabits ejaculated either once a week or once a day. J. Anim. Sci.. $15: 1221-1222,1956$.

23 - GROVE, D. Eine knunstliche scheide zur Gewinnung des 5 ames vom Kaninchenbock. IAn artificial vagina for collection semen from the male rabbit/. Dtsh. tierarztl. wschr., 70:492-494, 1953.

24 - HOOGEnKAMP, H. Het ejacula ate van de konijne ram. IEjaculate of the male rabbit/. T. Diergeneesk. $100: 592-596,1975$.

25 - HUHN, J. Einiges Über Gewinnung und Beurteilung des Kaninchenspermas. I collection and avaluation of rabbit semenl. Berl. Manch. tierârztl. Wschr., 65:243-247, 1952 .

26 - HUIZAR, M.E. Über den einfluss von Fotterungsfaktorem auf die Sameneigenschaften von Kaninctien. IEffect of nutritional factars on the semen characters of rabbits/. Hannover, 1974. 81p. IInaugural dissertation - Tierarztliche Hochschulel.

27 - KILHSTROM, J.E.; CARLSSON, R.; LAR5SON, M. A simple method for automatic couting of rabbits spermatozoa. J. Reprod. Fertil., $43: 371-372,1975$.

28 - KILHSTROM, J.E. \& FJELISTRÖM, D. Automatic counting of spermatozoa in rabbits semen. J. Reprod. Fertil., 14: $155-157,1967$.

29 - KIRTON, K.T.; DESJARDINS, C.; HAFS, H.D. Levels of some normal constituints of pabbit semen during repetitive ejaculation. Fertil. and Steril., 17:204-211, 1966.

30 - KREsteV, H.; RADEV, G.; DRNOU, D. IThe spermiogram. soermatozoal reserves and soermatozoal transport in the epicidymis of the rabbit/. Naueh. Tr. vissh. selskostop. Inst.
Georgi Dimitrov Zootekh. Fak. $13: 135-159,1963$.

31 - LAGERLöF, N. Morphologishe untersuchungen über veranderungen im spermabild und in den hoden bei bullen mit verminderter oder aufgehobener fertilitat. Acta pathol. mierobiol. scand. (suppl. 19): $1-254,1934$.

32 - MACARI, M.; MACHADO, C.R.; DONINI, C.A.; MARCHIORI, A.I.; GARCIA, J.M.; FAGLIRRI, J.J.; CARREGAL, R.D.; 5OARES, C.C. Caracterlsticas bioquimicas dos primeiros ejaculados de coelho. Cientlfica, Jaboticabal, 5:213-216, 1977 .

33 - MACARI, M. \& MACHADO, C.R. Sexual maturity in rabbits defined by the physical and chemica! characteristics of the semen. Lab. Anim., 12:37-39, 1978 .

34 - MACEDO, R.P. Estudo de algumas caracterlsticas do semen de coelho Oryctolagus cuniculus (Linnaeus, 1758). 530 Paulo, 1979, 47p. TTese de mestrado - Faculdade de Medicina Veterinaria e Zootecnia da Universidade de 5 ro Paulol.

35 - MRCEDO, A.P.; MIGUEL, D.; MUCCIDLD, R.G.; BRRNABE, R.C. Estudo de algumas caracteristicas do semen de coelho - Dryetolagus cuniculus (Linnaeus, 1758). Rev. Fac. Med. Vet. Zootec. Univ. 5ăo Paulo, 19: 139-151, 1982 .

36 - MACIRONE, C. \& WRLTON, R. Fecundity of male rabbits as determined by "dummy matings". J. Agric. Sci., $28: 122-134,1938$.

37 - MACMILLAN, K.L. \& HAFS, H.D. Semen output of rabbits ejaculated after varying sexual preparation. Proc. Soc. exp. Biol., New York, $125: 1278-1281,1967$.

38 - MATZKE, P. Ein Bejtrag zur Technik der künstlichen Besamung beim Kanichen. IA contribuition to the technique of artificial insemination in the rabbit/. Dtsch. tierarztl. Wschr., 63:143, 1956 apud HODGENKAMP,H., 24,1975, p.593.

39 - MIES FILHO, A. Reproducaro dos animais e inseminaca artificial. 5.ed. Porto Alegre, 5ulina, 1982. $2 v$.

40 - NUTTING

E.F.

Estimation spermatozoa numbers using 
micropore filtration technique. J. Reprod. Fertil., 20:341-344, 1969.

41 - D.FERRRL, G.L.M. \& MEACHAN, T.N. Relationship between $\mathrm{pH}$, other semen traits and fertility in rabbits. In: CONGRESS INTERNATIONAL DE REPRODUETION ANIMALE ET INSEMINATION ARTICIELLE, 6., Paris, 1958, V.2. P.1279-1282.

42 - PRUfLER

5.

Qualitativeund quantitative Untersuchunden der Spermigenese des Kanichens mit besonderer Berocksichtigung der histologischen Beurteilunsmethoden und Spermatozoen-Verluste im Hoden und Nebenhoden, 1968 apud HUIZRR, M.B., 26, p. 65 .

43 - SCHULZE, K. Beitrag zur morphologie der kaninchenspermien. IContribution to the morphology of rabbit spermatozoal/. Berl. Moneh. tiergrzt. Wschr., 77:445-447. 1964 .

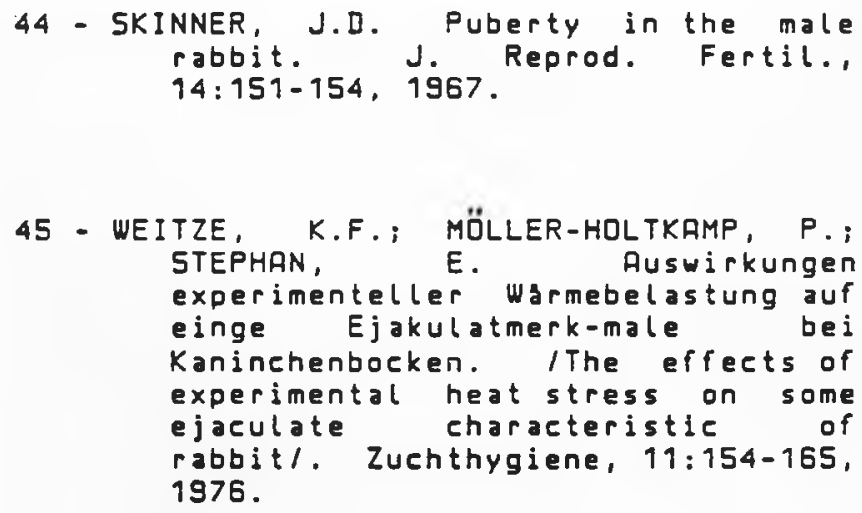

45 - WEITZE, K.F.; MÖLLER-HOLTKAMP, P.; STEPHAN, E. Auswirkungen experimenteller Warmebelastung auf einge Ejakulatmerk-male bei Kaninchenbocken. IThe effects of experimental heat stress on some ejaculate characteristic of rabbit/. Zuchthygiene, 11:154-165, 1976.

46 - WHITE, I.G. Biochemical aspects of mammalian semen. Anim. Breed. Abstr., 26:109-123, 1958.

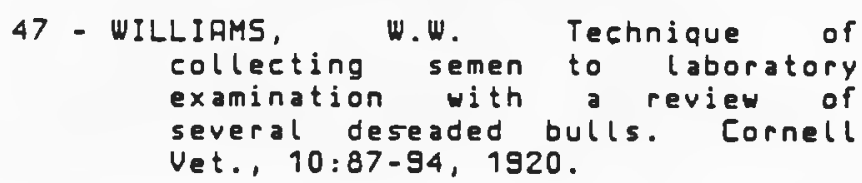

Rev.Fac.Med.vet.Zootec. Univ.S.Paulo, 23(1):55-67, 1986. 